\title{
Body composition with dual energy X-ray absorptiometry: from basics to new tools
}

\author{
Carmelo Messina ${ }^{1,2}$, Domenico Albano ${ }^{1,3}$, Salvatore Gitto ${ }^{2}$, Laura Tofanelli ${ }^{4}$, Alberto Bazzocchi ${ }^{5}$, \\ Fabio Massimo Ulivieri ${ }^{6}$, Giuseppe Guglielmi ${ }^{7,8}$, Luca Maria Sconfienza ${ }^{1,2}$
}

${ }^{1}$ IRCCS Istituto Ortopedico Galeazzi, Via Riccardo Galeazzi 4, 20161 Milano, Italy; ${ }^{2}$ Dipartimento di Scienze Biomediche per la Salute, Università degli Studi di Milano, Via Luigi Mangiagalli 31, 20133, Milano, Italy; ${ }^{3}$ Sezione di Scienze Radiologiche, Dipartimento di Biomedicina, Neuroscienze e Diagnostica Avanzata, Università degli Studi di Palermo, 90127, Palermo, Italy; ${ }^{4}$ Scuola di Specializzazione in Radiodiagnostica, Università degli Studi di Milano, Via Festa del Perdono 7, 20122 Milano, Italy; ${ }^{5}$ Diagnostic and Interventional Radiology, IRCCS Istituto Ortopedico Rizzoli, Via G. C. Pupilli 1, 40136, Bologna, Italy; ${ }^{6}$ Bone Metabolic Unit, Fondazione IRCCS Ca' Granda Ospedale Maggiore Policlinico. Medicina Nucleare, Via Francesco Sforza 35, 20122, Milano, Italy; ${ }^{7}$ Department of Radiology, University of Foggia, Viale Luigi Pinto 1, 71100, Foggia, Italy; ${ }^{8}$ Department of Radiology, Scientific Institute "Casa Sollievo della Sofferenza” Hospital, San Giovanni Rotondo, Foggia, Italy

Correspondence to: Dr. Carmelo Messina, MD. Via Riccardo Galeazzi, 20161 Milan, Italy. Email: carmelo.messina@unimi.it.

\begin{abstract}
Dual-energy X-ray absorptiometry (DXA) in nowadays considered one of the most versatile imaging techniques for the evaluation of metabolic bone disorders such as osteoporosis, sarcopenia and obesity. The advantages of DXA over other imaging techniques are the very low radiation dose, its accuracy and simplicity of use. In addition, fat mass (FM) and lean mass (LM) values by DXA shows very good accuracy compared to that of computed tomography and magnetic resonance imaging. In this review we will explain the technical working principles of body composition with DXA, together with the possible limitations and pitfalls that should be avoided in daily routine to produce high-quality DXA examinations. We will also cover the current clinical practical application of whole body DXA values, with particular emphasis on the use of LM indices in the diagnostic workup of reduced muscle mass, sarcopenia and osteosarcopenic obesity according to the most recent guidelines. The possible use of adipose indices will be considered, such as the fat mass index (FMI) or the android/gynoid ratio, as well as lipodystrophy indices and the evaluation of visceral adipose tissue (VAT). Whenever available, we will provide possible cut-off diagnostic values for each of these LM and FM indices, according to current literature and guidelines.
\end{abstract}

Keywords: Dual-energy X-ray absorptiometry (DXA); sarcopenia; obesity; fat mass index

Submitted May 03, 2019. Accepted for publication Feb 03, 2020.

doi: $10.21037 /$ qims.2020.03.02

View this article at: http://dx.doi.org/10.21037/qims.2020.03.02

\section{Introduction}

In 2017, dual energy X-ray absorptiometry (DXA) celebrated its $30^{\text {th }}$ anniversary (1). Since the initial development of this technique as the successor of dual photon absorptiometry, DXA technology assisted to continuous technological innovations and is now widely considered as the standard of reference in assessing bone mineral density (BMD) and fracture risk (1). At the same time, technology improvements opened the use of DXA to new applications, offering the possibility of evaluating several musculoskeletal parameters other than BMD. Body composition (BC) measurements have been early available after DXA discovery, despite this technique has been underutilized compared to BMD measurements (2). This paper aims to describe the principle of DXA science, to review the technical principles beyond $\mathrm{BC}$ measurements, and provide a summary of guidelines of clinical applications of soft tissue. 


\section{DXA: technical aspects}

The physical principle beyond DXA is that of X-ray transmission and attenuation thorough human body at two different energy levels (3). Basically, radiation energies emitted by DXA machines are variably attenuated based on both density and thickness of anatomical structures and tissues, as well as the intensity of emitted energy. Two aspects should be considered. First, the principle of X-ray attenuation relies on the fact that the higher the photon energy, the lowest the attenuation. Thus, the X-ray beam with lower energy will be more attenuated by tissues, with fewer photons passing through. Also, the density of material has to be taken in account, as high-density tissues (such as bone) attenuate the X-ray beam more than lowdensity tissues (such as soft tissues) (4). Based on this, DXA produces the so-called $\mathrm{R}$-value, which is the ratio between the attenuation coefficients at the two energy levels. This difference is specific for each tissue: while R-value is constant for bone and fat in all individuals, it varies for soft tissue as it depends on the patients' composition. For example, if a subject has a high fat percentage, the R-value will be lower compared to a subject with a high lean mass percentage (5).

DXA is capable to define the composition of human body by discriminating three different compartments, based on their specific X-ray attenuation properties: bone mineral content (BMC), lipid (triglycerides, phospholipid membranes, etc.) which is the so-called "fat mass" (FM), and lipid-free soft tissue which is the "lean mass" (LM). Of note, lipid-free soft tissue value represents the overall sum of overall body water, soft tissue mineral mass, protein, etc. (6). Based on this, the evaluation of DXA is typically considered a three-compartment model, although this technique is not capable to directly estimate all the three different components. In fact, about $40-45 \%$ of the total area of a DXA scan is composed of bone. In this area, DXA is only capable to differentiate between bone and the global amount of soft tissue, which includes both FM and LM. Pixels that are located adjacent to bone, which contain soft tissue only, are used to calculate the exact amount of FM and LM quantity $(3,7)$.

With regards to the source of X-ray emission, we assisted to a technological evolution with the transition from pencil-beam densitometers to fan-beam densitometers. The characteristic of pencil-beam densitometers is that they emit a single rectilinear highly collimated beam of $\mathrm{X}$-rays, coupled with a single detector (4). Despite offering measurements with higher precision compared to fan-beam, these densitometers need longer time to scan the wholebody surface. On the contrary, fan-beam technology uses a fan-shaped beam, which is coupled with multiple detectors, allowing shorter scan times with better image resolutions. The first fan beam scan was introduced by Hologic in 1993 with the QDR-2000 densitometer, while the first "narrow" fan beam scan was the GE Lunar Prodigy which was produced in 2003. Currently, Hologic Inc. (Bedford, MA, USA) and GE-Lunar Inc. (Madison, WI, USA) lead the market of densitometer manufacturers, both having recently introduced new high-resolution machines with several technical advancements (iDXA densitometer for GE Lunar, Horizon densitometer for Hologic) (7).

In general, the radiation dose of patients performing DXA is considered negligible (8). Exposure to ionizing radiation is frequently quantified as effective dose, which is expressed in sieverts ( $\mathrm{Sv}$ ) and is obtained by combining the information about the absorbed doses to the exposed tissue/organs and the relative radiation risk assigned to these tissue/organs (9). Effective dose is especially useful to compare different ionizing radiation sources and with natural background radiation. Performing a whole-body DXA scan with the last generation of densitometer expose patients to about $4-5 \mu \mathrm{Sv}$, which is even lower than the natural background dose (6.7 $\mu S_{v}$ per day) (3). As an example, the effective dose of a computed tomography head scan is reported to be around $2 \mathrm{mSv}$, which is comparable to 8-months exposure to natural background radiation (9). Radiation dose from DXA depends on different parameters, such as tube potential $(\mathrm{kVp})$ and tube current $(\mathrm{mA})$, size of the patient, number of images, imaging speed and the length of the scan. The operator can control a few of these parameters during whole body DXA. With regards to contraindications, the International Society of Clinical Densitometry (ISCD) considers pregnancy as the only relative contraindication to whole body DXA, due to the lack of justification to X-ray exposure (10).

\section{Comparison between DXA and other body composition imaging techniques}

Currently, magnetic resonance imaging (MRI) and computed tomography (CT) are considered the gold standard techniques for the assessment of muscle mass/ muscle quantity $(11,12)$. The reason is that both techniques are cross-sectional and allow for precise evaluation of compartmental BC at different regions of the body (skin, 
muscle, subcutaneous and visceral adipose tissue) (13). From a practical point of view, while DXA values of FM relate to the overall "chemical compartment" of triglycerides, CT and MRI measures of adipose tissue correspond to a specific "anatomical compartment", which contains adipocytes but also other constitutes such as collagenous fibres, fibroblasts, capillaries (3). As a consequence, FM measured by DXA is somewhat lower than the corresponding values measured by cross-sectional imaging. On the other side, LM measured by DXA is somewhat higher than that measured by MRI and CT, as it includes body proteins together with body water, non-fat lipids, carbohydrates, soft tissue minerals (14).

Nevertheless, there are still several constraints that limit the widespread use of CT and MRI in daily routine. First of all, both techniques are costly and require trained professionals to perform and analyse these exams, which typically need specific post-processing (15). Other limitations are the compliance of patients, such as claustrophobia or subject motion with poor image quality (especially for MRI, which also has contraindications), and the higher dose of radiation provided by CT with respect to DXA (11). More importantly, there is still a lack of evidence about the specific cut-off values that should be used for defining "low muscle mass" on data provided by CT and MRI (16).

On the contrary, DXA is a low-dose radiation technique, which is generally reported to be more accessible and comfortable for the patients, that can easily provide several $\mathrm{BC}$ indices in a few minutes. In addition to this, despite the above-mentioned differences between DXA and crosssectional techniques measurements, several studies have found a very good correlation between FM measured by DXA and CT/MRI (3). In particular, a study by Chen et al. compared DXA-derived measurements of lean mass with skeletal muscle mass measured with MRI, showing a very high correlation both for the whole body lean mass $(r=0.94)$ and leg region $(r=0.91)(14)$. Another study by the Japanese group of Midorikawa et al. confirmed these good correlations also in the paediatric population of prepubertal boys and girls (17). A study form Bredella et al. compared CT and DXA, providing similar values of correlation in BC measurements ( $r=0.77-0.95)$; nevertheless, this study warned about the fact that these values are lower in markedly obese women, thus DXA may not provide the same accuracy in that population (18).

For all these reasons, there is a general consensus about the fact that DXA should be considered the technique of reference for the assessment in clinical practice of BC
$(16,19,20)$. More specifically, the revised version of the European Working Group on Sarcopenia in Older People (EWGSOP) guideline suggests using DXA for detecting "low muscle mass" in clinical practice, while considers more appropriate the use of CT and MRI in the research setting (16).

\section{Performing a whole body DXA scan: practical points}

The accuracy of LM and FM values produced by DXA strictly depends on the multi-step process of whole-body scan execution. This multi-step process is composed of three parts, each of them of great importance: subject preparation, subject positioning, and scan post-processing. Figure 1 shows an example of two properly positioned and analysed DXA BC scan.

Proper patient preparation is crucial for maintaining the biological variability of LM and FM measurements as low as possible. It is well known that BC evaluation is influenced by hydration and digestive tract content. Meals have been associated with an increase of trunk and whole body lean mass values (21). Exercise is another condition that can increase $L M$ values at limbs with a decrease of trunk $L M$, due to fluid movement from trunk to periphery (22). Body FM seems less affected by meals and exercise, but can vary according to the hydration status. In fact, the technology of DXA is based on the assumption that the hydration of fatfree tissue remains constant (at around $73 \%$ ). Thus, FM may vary in cases of severe overhydration due to conditions such as ascites or edema, with a percentage that has been reported in simulated studies of about $1 \%$ when hydration changes of $5 \%(23)$. As a general rule, the good practice would be to measure BC with DXA always in standardized conditions in terms of activities, food intake, hour of the day. It is reported that the best condition to be reproducible is to scan the patient after an overnight fast, so that "real" changes can be easily detected (22).

Patient positioning is another critical aspect, as errors in this step lead to unavoidable difficulties in the subsequent analysis process. The first step is to ensure that subjects receiving the DXA remove all external metal that may interfere and attenuate the X-ray beam, thus creating artifacts. Patients should be positioned in the center of the scan with the use of reference lines on the scan table (central line and border limit lines). ISCD official positions suggest the following method for positioning the patient that should be used whenever possible $(24,25)$. The upper 

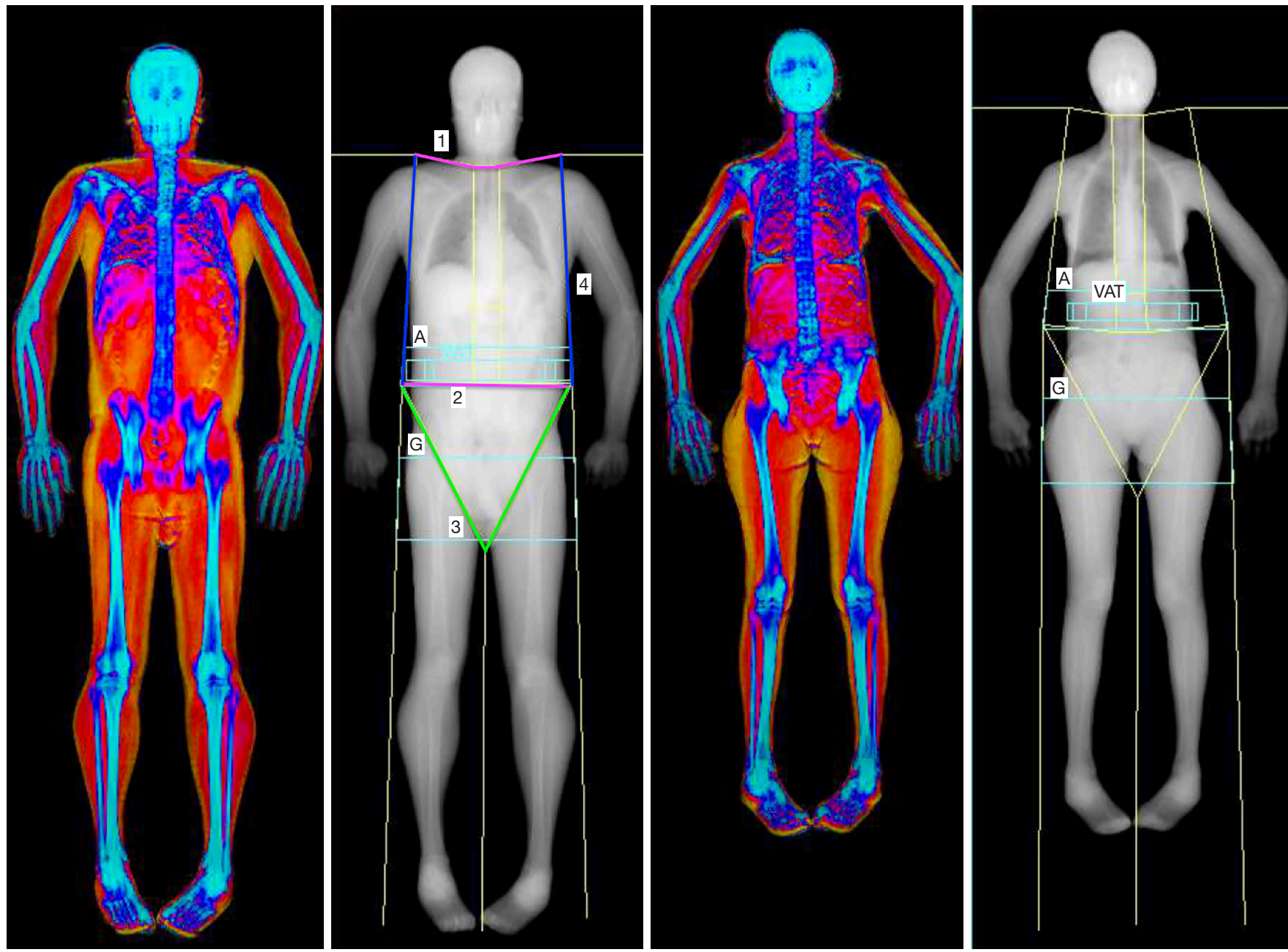

Figure 1 Two examples of proper positioned and post-processed whole body DXA scan, according to Hologic manufacturer guidelines. The visual comparison of a young man (first two images on the left side) with a middle-aged woman (the last two images on the right side) clearly depict differences in fat distribution (represented in yellow), with the woman showing the typical gynoid distribution around the hips and thighs. Head line (number 1) is placed below the jaw; pelvis line (number 2) has to be placed just above the iliac crests; two groin lines (number 3, in green) have to bisect the femoral neck on each side; trunk lines (number 4, in blue) separate the arm regions from the trunk android regions, and they should pass through the gleno-humeral joints along each side of the trunk. A, android region; G, gynoid region; VAT, visceral adipose tissue region.

limbs should lie along the body, with hands palm down without any superimposition with the body. Feet should stay in a neutral or slightly internal rotated position, with residual space left between the lower limbs. Head should be positioned whit chin in neutral position, face up; pillows should not be used (6).

The last step relates to image analysis, which should be done according to the manufacturer's instructions. As a general rule, symmetry should be maintained between the left and right side when placing regional lines, in order to include on both sides the same amount of soft tissue and bone. The position of major lines is similar for both GE Lunar and Hologic densitometers: the upper horizontal line has to be placed just under the jaw, while the pelvis horizontal placement is just above the iliac crests. The vertical lines on both sides of the trunk should separate arms from the chest wall, while the vertical lines in the lower limbs have to be placed around the hip/leg. One last vertical line should be placed between legs to separate them $(4,24)$. See Figure 1 for a detailed explanation of DXA analysis. In 

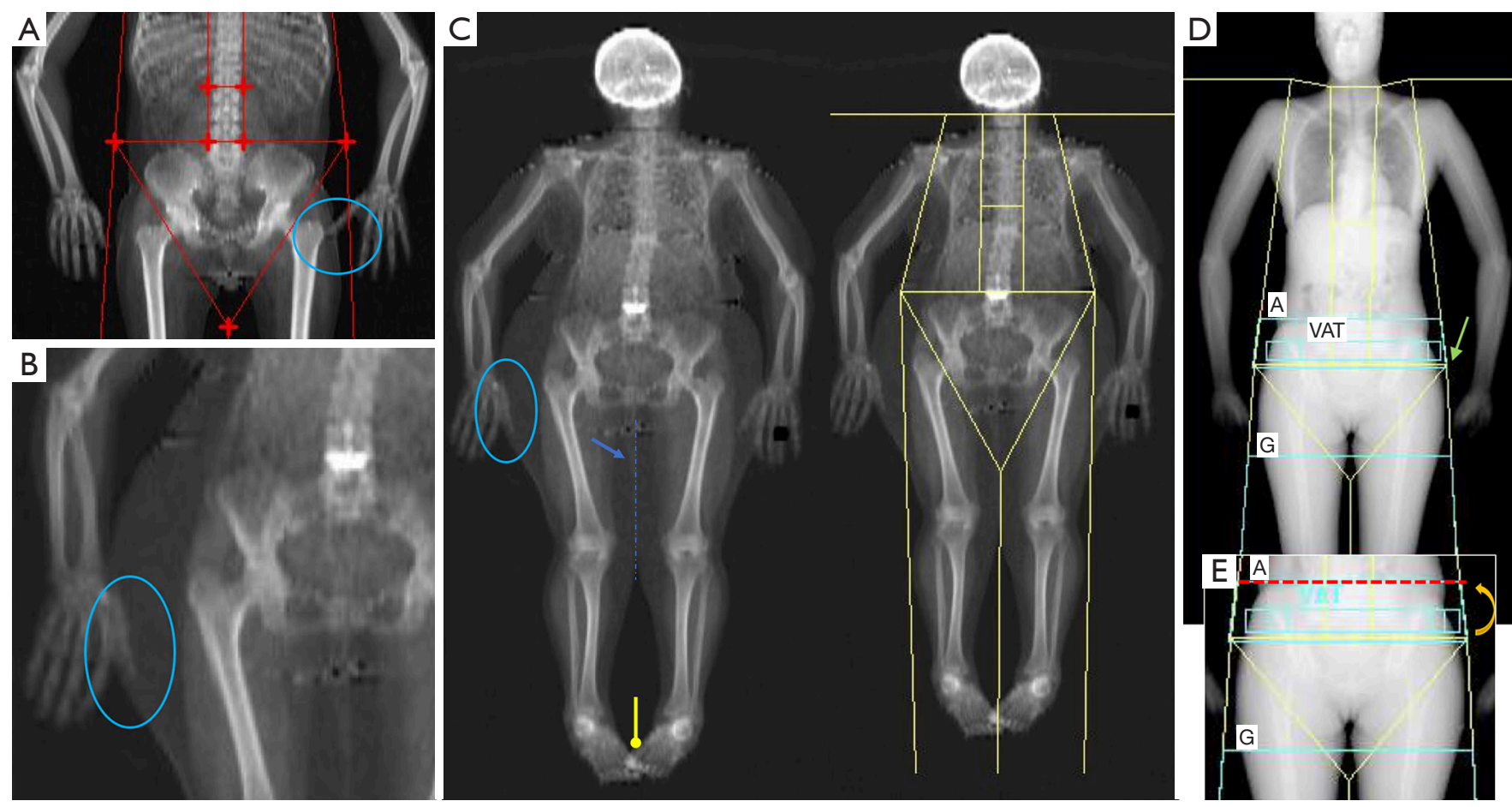

Figure 2 Examples of common positioning and postprocessing artifacts that may limit the accuracy of whole body DXA scan. (A,B) Two examples of superimposition between fingers and leg fat tissue (see the blue circles). (C) Overweight patients in which positioning may result complicated: in this case there is an overall superimposition between several anatomic regions such as fingers with subcutaneous fat (blue circle), foots (yellow line) and legs (blue arrow). The latter may create problems in separating the fat mass content between the two legs (dashed blue line). (D) Inaccurate placement of pelvis line (green arrow), which is located just above both femoral heads; this create problems in the correct identification of android and gynoid regions, which is typically done automatically by the software. (E) Image shows the correct placement of the pelvic line (dashed red line), after image correction (indicated by curved arrow).

addition, Figure 2 shows some examples of inaccuracies in positioning and analysing a whole body DXA scan.

\section{Can we use DXA for the diagnosis of sarcopenia? Current indications}

Sarcopenia is a disease typically associated with advancing age, characterized by a progressive and generalized loss of skeletal muscle mass and strength (13,26-28). The disease was typically considered a geriatric syndrome, but it is now recognized as an independent disease with its own code in the tenth revision of the International Classification of Disease (ICD-10-CM, code = M62.84) (29). There is increasing awareness of the medical community for this condition, as it has been estimated a consistent increase of the prevalence of sarcopenia due to advancing age, as well as for its association with several negative outcomes such as physical disability, falls, hospitalization (30-32). The disease is costly to healthcare systems, as it has been shown that the cost of care during hospitalization is increased in subject with sarcopenia (33).

In 2018, the EWGSOP revised its operational definition of sarcopenia, giving primary importance to muscle strength as the most important parameter for predicting adverse outcomes (16). More practically, according to EWGSOP definition, a diagnosis of sarcopenia is probable when low muscle strength is detected. The diagnosis is then confirmed by the association with low muscle quantity/ quality. When also low physical performance is present, the diagnosis of sarcopenia is considered severe (16). Each of these parameters can be measured: muscle strength and physical performance are typically measured by means of different tests such as grip strength, chair stand, gait speed or the Short Physical Performance Battery; on the contrary, the evaluation of muscle quantity or quality needs the use of imaging or non-imaging techniques, such as DXA, MRI, CT or Bioelectrical impedance analysis (BIA) $(15,34,35)$. 
Among the different imaging techniques available to quantify muscle mass, DXA is currently favoured by clinicians and working groups (36). DXA is capable of determining regional and total body muscle quantity, providing both absolute values of LM as well as LM indices, such as the appendicular skeletal muscle mass (ASMM). Nevertheless, it has to be taken into account that muscle mass correlates to body size. As a consequence, values from DXA are typically adjusted to body parameters such as height ${ }^{2}$, weight, or body mass index (BMI) (37). The type of adjustment for the specific population is debated, but many guidelines suggest now the use of ASMM with height ${ }^{2}$ adjustment (ASMM/height ${ }^{2}$ ), which is also known as ASMM index (ASMMI) $(16,19,24)$. Several absolute thresholds of ALMI have been proposed over time, with the most recent update in 2018 by the EWGSOP2 suggesting to use the following values as lower cut-off points: ASMMI $<7.0 \mathrm{~kg} / \mathrm{m}^{2}$ for men and ASMMI $<6.0 \mathrm{~kg} / \mathrm{m}^{2}$ for women $(11,16)$. Alternatively, absolute values of ASMM can either be used, with the following lower cut-off points: ASMM $<20 \mathrm{~kg}$ for men, ASMM $<15 \mathrm{~kg}$ for women $(16,27)$.

It is very important to point out that the abovementioned values should not be used alone to diagnose "sarcopenia". In fact, such diagnosis needs the concurrent presence of low muscle strength detected at functional tests. So, it is encouraged to use the term "low muscle mass" in DXA reports in case of reduced ASMM/ASMMI values (24). In addition to this, the medical community is debating about the clinical value of combining the use of ASMMI and femoral neck BMD to predict fracture risk, similarly to what is commonly performed in the Fracture Risk Assessment Tool $\left(\right.$ FRAX $^{\circledR}$ ) (38). To date, literature evidence suggests that DXA-derived ASMMI has a limited role in the prediction of incident fractures (39). Despite the well-known link between bone and muscle, fracture is just one outcome resulting from sarcopenia, and ASMMI may have better value for identifying other adverse outcomes such as falls. In their editorial, Harvey concludes that the use of DXA-derived lean mass indices still does not provide additional risk information when $\mathrm{BMD}$ is also considered; further studies are warranted to understand the role of possible use of ASMMI in the FRAX. The possible introduction of newer DXA based tools for evaluating bone strain together with body composition may even provide additional information for fracture risk prediction (40).

A possible DXA drawback is related to the fact that densitometers of different brands may not give the same results $(36,41)$. A practical suggestion is to perform the follow-up scan always with the same densitometer, to remove such source of variability.

\section{Whole body DXA and adipose indices: how can we use them?}

Several adipose indices can be obtained with whole body DXA, some of them being similar to lean indices. These values help in providing additional data about whole body and regional FM distribution. Among them we will discuss the fat mass index (FMI), the Android/Gynoid (AG) ratio and lipodystrophy indices (\% fat Trunk/Leg, Trunk/limb fat mass ratio).

FMI is calculated as the total body fat mass with height ${ }^{2}$ adjustment. The use of FMI has been advocated to diagnose obesity, similarly to what done by BMI. The rationale of using FMI is based on the assumption that BMI, despite its ease of use, is not a direct adiposity measurement parameter (25). On the contrary, despite the use of FMI (which is a direct measurement of adiposity) has its rationale, there is an ongoing debate about what cut-off point should be used to better diagnose obesity. Figure 3 compares BMI thresholds to a list of FMI cut-off points proposed for defining obesity categories both for male and female subjects using FMI, according to the National Health and Nutrition Examination Survey (NHANES) reference values (42). Nevertheless, it is still unclear which associations exist between such thresholds and obesity related negative outcomes. Despite this, FMI has a clear advantage over BMI for the evaluation of obesity, as the latter does not take into account patients' lean mass.

Regarding obesity, several studies clearly showed that DXA measurements of FM (including FMI) are strongly correlated with negative cardiovascular and metabolic outcomes, independent of BMI $(44,45)$. A recent study from Vasan et al. evaluated the associations between DXA values and conventional anthropometry measurements of fat (waist and hip circumference), together with cardiovascular disease (CVD) risk markers (46). The result of this study confirmed that conventional anthropometry underestimated the associations of regional adiposity (subcutaneous and visceral fat) with diabetes and CVD risk markers.

Another parameter that can be used for evaluating the CVD risk is the AG fat mass ratio, which is analogue to the more commonly used anthropomorphic measurement of waist-to-hip ratio (47). DXA machines obtain this value as the ratio between the android and gynoid region of interest (ROI). Android ROI is typically defined as the region 


\begin{tabular}{|l|c|c|c|c|c|}
\hline \multirow{2}{*}{ Gender } & \multicolumn{5}{|c|}{ Fat mass index $\left(\mathrm{kg} / \mathrm{m}^{2}\right)$} \\
\cline { 2 - 6 } & Normal & Overweight & Obesity Class I & Obesity Class II & Obesity Class III \\
\hline Female subjects & $5-9$ & $>9-13$ & $>13-17$ & $>17-21$ & $>12-15$ \\
\hline Male subjects & $3-6$ & $>6-9$ & $>9-12$ & $>15$ \\
\hline & \multicolumn{5}{|c|}{ Body mass index $\left(\mathrm{kg} / \mathrm{m}^{2}\right)$} \\
\hline
\end{tabular}

Figure 3 Comparison between the values of DXA-based fat mass index (obtained as the ratio between fat mass/height ${ }^{2}$ ) and those of body mass index in the classifications for obesity categories. Fat mass index categories are obtained from NHANES reference values (42); body mass index categories from World Health Organization (WHO) (43).

between the last thoracic rib and the upper part of iliac wings. Gynoid ROI is located below the android ROI, and includes the gluteo-femoral region with an upper horizontal line placed caudally to the pelvis line, and a lower horizontal line identified by measuring twice the height of the android ROI (4). It has been shown that an increase in the android fat distribution (with values of AG ratio greater than 1) is associated with conditions such as dyslipidemia and insulin resistance, as well as other cardiovascular risk factors such as impaired glucose tolerance, hypercholesterolemia, hypertriglyceridemia, and hypertension (47-49). Unfortunately, as for FMI, despite the evidence of a strong correlation between the increasing amount of android fat and CVD risk, there is still no consensus on the possible cut-off points to be used for defining a specific high-risk patient.

Recent evidence of the possible concurrent presence of low BMD (osteopenia or osteoporosis), reduced muscle mass (sarcopenia), and increased adiposity (obesity) led the scientific community to propose a new term, the so-called "osteosarcopenic obesity" (50). As a matter of fact, DXA is currently the only technique which is capable of identifying all these conditions at the same time, by evaluating the presence of low ASMMI together with adipose indices (such as FMI) and BMD (with DXA performed at lumbar spine and femur). Figure 4 compares two subjects with reduced muscle mass according to ASMMI values, but with different percentage of fat mass as evaluated with FMI.

The use of trunk/limb FM ratio have been advocated to evaluate the fat redistribution in HIV patients treated with older regimens of antiretroviral agents, which were at risk of complications such as lipodystrophy and lipoatrophy (25). In fact, the association of lipodystrophy with agents such as zidovudine and stavudine has been extensively reported, being confirmed in a systematic review of randomized controlled trials (51). Nevertheless, the ISCD Reporting guideline of 2013 clearly specified that a consensus about the values to be accepted has never been reached (25). Recently in 2019 a study by Alikhani et al. evaluated the prevalence of lipodystrophy in HIV patients and the association to cumulative exposure of newer antiretroviral drugs agents (52). In this study, the DXA cut-off point to define lipodystrophy was the presence of trunk/limb fat ratio $\geq 1.5$. Surprisingly, lipodystrophy resulted still very common in HIV infected patients, being correlated with the duration of some new antiretroviral drugs such as raltegravir. Such results keep open the possibility to still use DXA for the evaluation of suspected lipodystrophy, and probably suggests additional investigations are required to understand the utility of such adipose indices.

\section{Visceral fat assessment with whole body DXA: technical aspects}

One of the most recent developments for whole body DXA is the possibility to evaluate visceral adipose tissue (VAT). This can be done with both GE and Hologic densitometers, thanks to new software which are called CoreScan ${ }^{\mathrm{TM}}$ for GE-Lunar and InnerCore ${ }^{\mathrm{TM}}$ for Hologic (4). This software firstly estimates the amount of subcutaneous fat (SAT) in the android ROI of DXA scan, by detecting the fat located on both sides of the abdominal cavity. This estimate of SAT is then subtracted from the total FM in the android ROI, thus providing the final amount of VAT. Figure 5 explains the working principle beyond VAT estimation by DXA. Measurements of VAT by DXA showed to be highly correlated with those obtained by CT ( $r=0.93)$ (53). DXA VAT measurements have several advantages over CT, first 

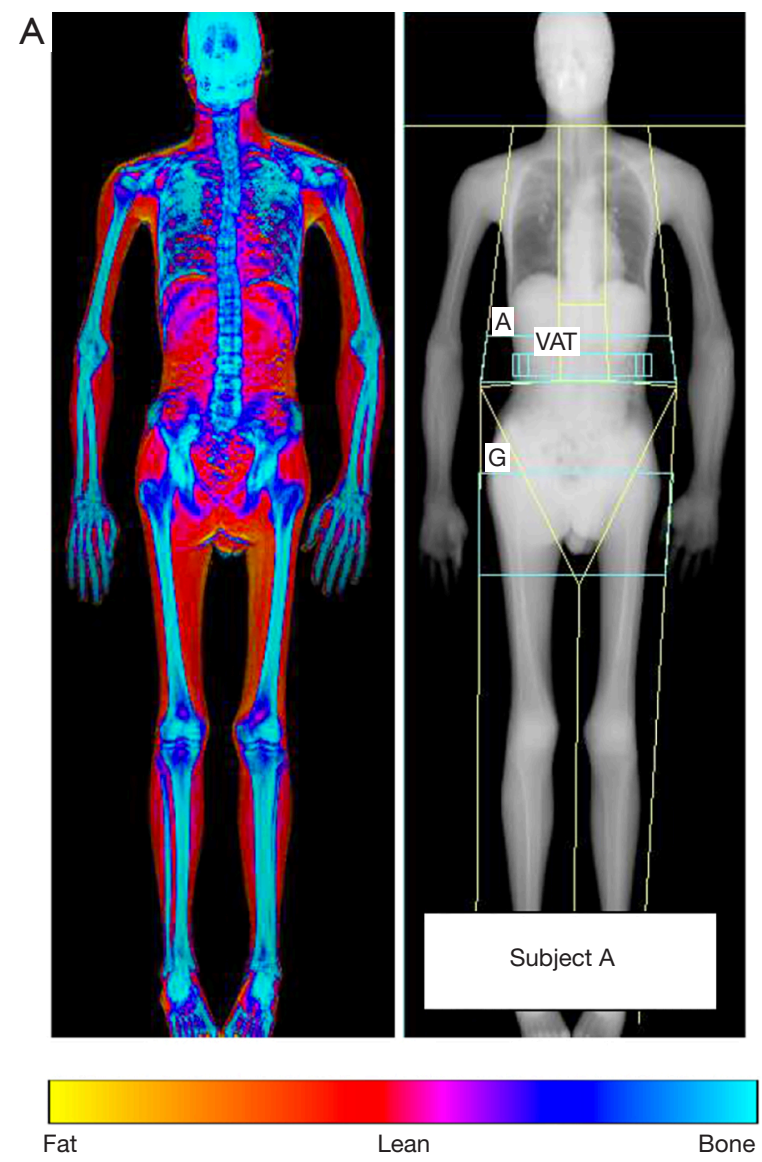

Adipose Indices

\begin{tabular}{|lccc|}
\hline Measure & Result & \multicolumn{2}{c|}{ Percentile } \\
& & YN & AM \\
Total Body \% Fat & 17.9 & 13 & 6 \\
Fat Mass/Height ${ }^{2}\left(\mathrm{~kg} / \mathrm{m}^{2}\right)$ & $2.82^{*}$ & 2 & 1 \\
Android/Gynoid Ratio & 0.78 & & \\
\% Fat Trunk/\% Fat Legs & 0.79 & 17 & 8 \\
Trunk/Limb Fat Mass Ratio & 0.85 & 19 & 10 \\
Est. VAT Mass $(\mathrm{g})$ & 169 & & \\
Est. VAT Volume $\left(\mathrm{cm}^{3}\right)$ & 183 & & \\
Est. VAT Area $\left(\mathrm{cm}^{2}\right)$ & 35.1 & & \\
\hline
\end{tabular}

Lean Indices

\begin{tabular}{|c|c|c|c|}
\hline \multirow[t]{2}{*}{ Measure } & \multirow[t]{2}{*}{ Result } & \multicolumn{2}{|c|}{ Percentile } \\
\hline & & YN & AM \\
\hline Lean/Height ${ }^{2}\left(\mathrm{~kg} / \mathrm{m}^{2}\right)$ & 12.3 & 1 & 1 \\
\hline Appen. Lean/Height ${ }^{2}\left(\mathrm{~kg} / \mathrm{m}^{2}\right)$ & 5.31 & 1 & 1 \\
\hline
\end{tabular}

B
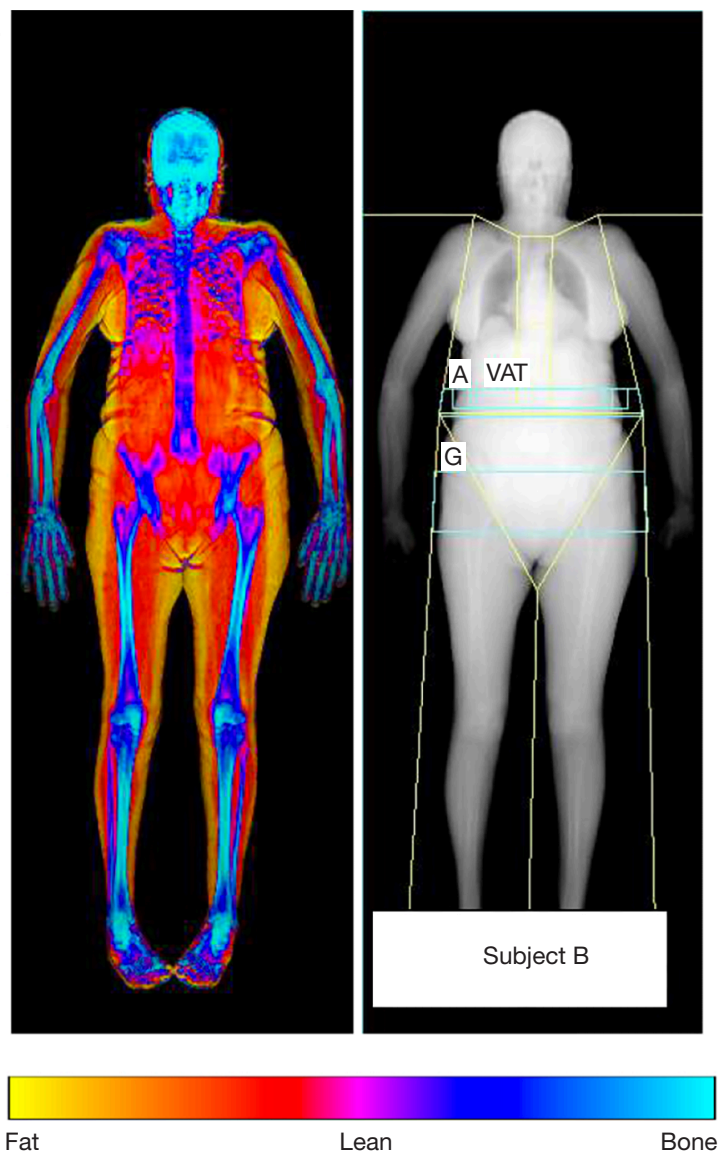

Adipose Indices

\begin{tabular}{|lccc|}
\hline Measure & Result & \multicolumn{3}{c|}{ Percentile } \\
& & YN & AM \\
Total Body \% Fat & 42.2 & 79 & 45 \\
Fat Mass/Height ${ }^{2}\left(\mathrm{~kg} / \mathrm{m}^{2}\right)$ & $11.1^{\star}$ & 69 & 44 \\
\hline Android/Gynoid Ratio & 1.06 & & \\
$\%$ Fat Trunk/\% Fat Legs & 0.83 & 61 & 34 \\
Trunk/Limb Fat Mass Ratio & 0.90 & 68 & 35 \\
Est. VAT Mass $(\mathrm{g})$ & 833 & & \\
Est. VAT Volume $\left(\mathrm{cm}^{3}\right)$ & 901 & & \\
Est. VAT Area $\left(\mathrm{cm}^{2}\right)$ & 173 & & \\
\hline
\end{tabular}

\section{Lean Indices}

\begin{tabular}{|lccc|}
\hline Measure & Result & \multicolumn{3}{c|}{ Percentile } \\
& & YN & AM \\
Lean/Height ${ }^{2}\left(\mathrm{~kg} / \mathrm{m}^{2}\right)$ & 14.6 & 40 & 42 \\
Appen. Lean/Height ${ }^{2}\left(\mathrm{~kg} / \mathrm{m}^{2}\right)$ & 5.86 & 27 & 41 \\
\hline
\end{tabular}

Figure 4 Comparison between two subjects with low appendicular skeletal muscle mass index (ASMMI) values (red arrow) and different values of fat mass index (FMI, asterisks). Subject A is a young male with long-standing facioscapulohumeral muscular dystrophy, presenting with both reduced ASMMI $\left(5.31 \mathrm{~kg} / \mathrm{m}^{2}\right.$, normal values for men $>7.0 \mathrm{~kg} / \mathrm{m}^{2}$ ) and very low values of FMI (normal values for men between 3 and $6 \mathrm{~kg} / \mathrm{m}^{2}$ ), suggesting the concurrent presence of reduced muscle mass and underweight. Subject B is a middle-aged woman with reduced muscle mass (ASMMI $=5.86 \mathrm{~kg} / \mathrm{m}^{2}$, normal values for women $>6.0 \mathrm{~kg} / \mathrm{m}^{2}$ ) but with high values of FMI, a condition which may raise the suspect for sarcopenic obesity. 

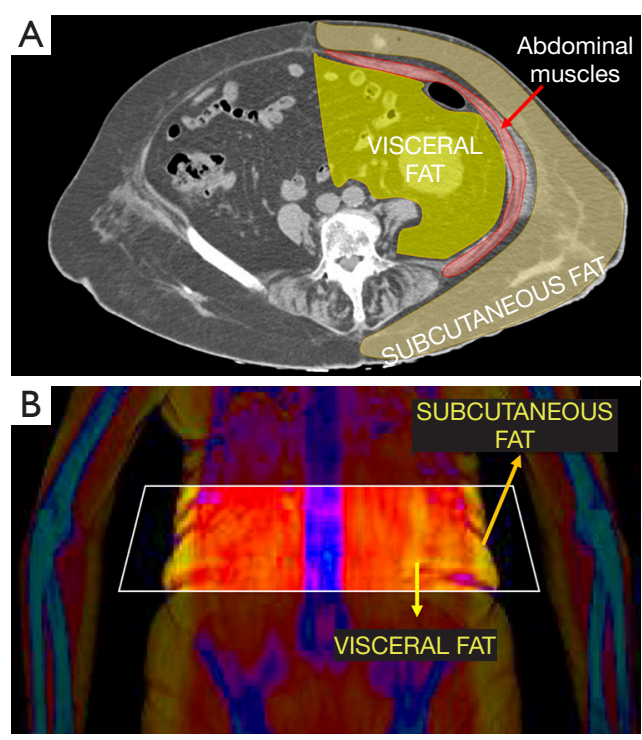

Figure 5 Description of the working principle beyond visceral adipose tissue (VAT) estimation with DXA. Image A shows a cross sectional computed tomography image of the abdomen, which depicts the intra-abdominal visceral fat that is separated from subcutaneous fat (SF) by means of abdominal muscles lean mass. Image B shows the corresponding DXA example of VAT/SF visualization: the estimation VAT is obtained by subtracting SAT from the total FM in the android region of interest.

and foremost related to lower radiation dose provided to patients.

The clinical importance of VAT is widely recognized, as it has been showed that VAT is a better predictor of mortality than SAT or AG ratio (54). Thus, it is possible that VAT will replace the AG ratio as a risk factor for the assessment of CVD risk with DXA (25). It is important to consider that even subjects with normal values of BMI may have an increased amount of VAT accumulation, thus being at higher risk than that estimated by only conventional anthropometry measurements (46).

Regarding thresholds, VAT is typically expressed as a real measurement in $\mathrm{cm}^{2}$. Cross-sectional studies suggested that VAT levels exceeding $100-110 \mathrm{~cm}^{2}$ were associated with adverse metabolic profile in women (55). On CT measurements of VAT, Pickhardt et al. found that a threshold of $70 \mathrm{~cm}^{2}$ yielded a sensitivity, specificity, and accuracy of $83.7 \%, 80.0 \%$, and $81.3 \%$, respectively, for metabolic syndrome in women (56). Another paper suggests specific cut-off points of $100 \mathrm{~cm}^{2}$ for considering a subject at increased "metabolic risk", while a VAT higher than
$160 \mathrm{~cm}^{2}$ is associated with high metabolic risk (57).

\section{Conclusions}

The analysis of body composition with DXA is becoming more and more popular among physicians, as DXA has the potentiality to evaluate LM and FM with an accuracy that is close to that of CT and MRI, but with some advantages compared to cross-sectional techniques. As a consequence, whole body DXA is increasingly used for different potential applications, such as the investigation of low lean mass in the setting of suspected sarcopenia. The possible combination of LM and FM values with that of BMD obtained with DXA at central site open the possibility for a more comprehensive evaluation of osteometabolic disorders, such as osteosarcopenia and sarcopenic obesity. There is still a lot that has to be done, and further research studies are warranted to provide clear cutoff values for adipose indices in cardiovascular disease, as well as to understand the role of ASMMI in the possible prediction of fracture risk in patients with sarcopenia.

\section{Acknowledgments}

Funding: None.

\section{Footnote}

Provenance and Peer Review: With the arrangement by the Guest Editors and the editorial office, this article has been reviewed by external peers.

Conflicts of Interest: All authors have completed the ICMJE uniform disclosure form (available at http://dx.doi. org/10.21037/qims.2020.03.02). The special issue "Body Composition Imaging" was commissioned by the editorial office without any funding or sponsorship. GG served as the unpaid Guest Editor of the special issue and serves as an unpaid editorial board member of Quantitative Imaging in Medicine and Surgery. AB served as the unpaid Guest Editor of the special issue. The authors have no other conflicts of interest to declare.

Open Access Statement: This is an Open Access article distributed in accordance with the Creative Commons Attribution-NonCommercial-NoDerivs 4.0 International License (CC BY-NC-ND 4.0), which permits the noncommercial replication and distribution of the article with 
the strict proviso that no changes or edits are made and the original work is properly cited (including links to both the formal publication through the relevant DOI and the license). See: https://creativecommons.org/licenses/by-nc-nd/4.0/.

\section{References}

1. Glüer CC. 30 years of DXA technology innovations. Bone 2017;104:7-12.

2. Shepherd JA, Ng BK, Sommer MJ, Heymsfield SB. Body composition by DXA. Bone 2017;104:101-5.

3. Guglielmi G, Ponti F, Agostini M, Amadori M, Battista G, Bazzocchi A. The role of DXA in sarcopenia. Aging Clin Exp Res 2016;28:1047-60.

4. Bazzocchi A, Ponti F, Albisinni U, Battista G, Guglielmi G. DXA: Technical aspects and application. Eur J Radiol 2016;85:1481-92.

5. Laskey MA. Dual-energy X-ray absorptiometry and body composition. Nutrition 1996;12:45-51.

6. Dual Energy X Ray Absorptiometry for Bone Mineral Density and Body Composition Assessment, Human Health Series No. 15. Vienna: International Atomic Energy Agency, 2011.

7. Toombs RJ, Ducher G, Shepherd JA, De Souza MJ. The impact of recent technological advances on the trueness and precision of DXA to assess body composition. Obesity (Silver Spring) 2012;20:30-9.

8. Damilakis J, Adams JE, Guglielmi G, Link TM. Radiation exposure in X-ray-based imaging techniques used in osteoporosis. Eur Radiol 2010;20:2707-14.

9. Mettler FA, Huda W, Yoshizumi TT, Mahesh M. Effective doses in radiology and diagnostic nuclear medicine: a catalog. Radiology 2008;248:254-63.

10. Kendler DL, Borges JL, Fielding RA, Itabashi A, Krueger D, Mulligan K, Camargos BM, Sabowitz B, Wu CH, Yu EW, Shepherd J. The Official Positions of the International Society for Clinical Densitometry: Indications of Use and Reporting of DXA for Body Composition. J Clin Densitom 2013;16:496-507.

11. Beaudart $\mathrm{C}$, McCloskey $\mathrm{E}$, Bruyère $\mathrm{O}$, Cesari $\mathrm{M}$, Rolland Y, Rizzoli R, Araujo de Carvalho I, Amuthavalli Thiyagarajan J, Bautmans I, Bertière MC, Brandi ML, AlDaghri NM, Burlet N, Cavalier E, Cerreta F, Cherubini A, Fielding R, Gielen E, Landi F, Petermans J, Reginster JY, Visser M, Kanis J, Cooper C. Sarcopenia in daily practice: assessment and management. BMC Geriatr 2016;16:170.

12. Albano D, Messina C, Vitale J, Sconfienza LM. Imaging of sarcopenia: old evidence and new insights. Eur Radiol
2020;30:2199-208.

13. Messina C, Maffi G, Vitale JA, Ulivieri FM, Guglielmi G, Sconfenza LM. Diagnostic imaging of osteoporosis and sarcopenia: A narrative review. Quant Imaging Med Surg 2018;8:86-99.

14. Chen Z, Wang Z, Lohman T, Heymsfield SB, Outwater E, Nicholas JS, et al. Dual-energy X-ray absorptiometry is a valid tool for assessing skeletal muscle mass in older women. J Nutr 2007;137:2775-80.

15. Codari M, Zanardo M, di Sabato ME, Nocerino E, Messina C, Sconfienza LM, Sardanelli F. MRI-Derived Biomarkers Related to Sarcopenia: A Systematic Review. J Magn Reson Imaging 2020;51:1117-27.

16. Cruz-Jentoft AJ, Bahat G, Bauer J, Boirie Y, Bruyère O, Cederholm T, Cooper C, Landi F, Rolland Y, Sayer AA, Schneider SM, Sieber CC, Topinkova E, Vandewoude M, Visser M, Zamboni M; Writing Group for the European Working Group on Sarcopenia in Older People 2 (EWGSOP2), and the Extended Group for EWGSOP2. Sarcopenia: revised European consensus on definition and diagnosis. Age Ageing 2019;48:16-31.

17. Midorikawa T, Ohta M, Hikihara Y, Torii S, Sakamoto S. Predicting skeletal muscle mass from dual-energy X-ray absorptiometry in Japanese prepubertal children. Eur J Clin Nutr 2017;71:1218-22.

18. Bredella MA, Ghomi RH, Thomas BJ, Torriani M, Brick DJ, Gerweck AV, Misra M, Klibanski A, Miller KK. Comparison of DXA and CT in the Assessment of Body Composition in Premenopausal Women With Obesity and Anorexia Nervosa. Obesity (Silver Spring) 2010;18:2227-33.

19. Chen LK, Liu LK, Woo J, Assantachai P, Auyeung TW, Bahyah KS, Chou MY, Chen LY, Hsu PS, Krairit O, Lee JS, Lee WJ, Lee Y, Liang CK, Limpawattana P, Lin CS, Peng LN, Satake S, Suzuki T, Won CW, Wu CH, Wu SN, Zhang T, Zeng P, Akishita M, Arai H. Sarcopenia in Asia: consensus report of the Asian Working Group for Sarcopenia. J Am Med Dir Assoc 2014;15:95-101.

20. Messina C, Monaco CG, Ulivieri FM, Sardanelli F, Sconfienza LM. Dual-energy X-ray absorptiometry body composition in patients with secondary osteoporosis. Eur J Radiol 2016;85:1493-8.

21. Horber FF, Thomi F, Casez JP, Fonteille J, Jaeger P. Impact of hydration status on body composition as measured by dual energy X-ray absorptiometry in normal volunteers and patients on haemodialysis. Br J Radiol 1992;65:895-900.

22. Nana A, Slater GJ, Hopkins WG, Burke LM. Effects of 
Daily Activities on Dual-Energy X-ray Absorptiometry Measurements of Body Composition in Active People. Med Sci Sports Exerc 2012;44:180-9.

23. Pietrobelli A, Wang Z, Formica C, Heymsfield SB. Dual-energy X-ray absorptiometry: fat estimation errors due to variation in soft tissue hydration. Am J Physiol 1998;274:E808-16.

24. Shepherd JA, Baim S, Bilezikian JP, Schousboe JT. Executive summary of the 2013 International Society for Clinical Densitometry Position Development Conference on Body Composition. J Clin Densitom 2013;16:489-95.

25. Petak S, Barbu CG, Yu EW, Fielding R, Mulligan K, Sabowitz B, Wu CH, Shepherd JA. The Official Positions of the International Society for Clinical Densitometry: Body Composition Analysis Reporting. J Clin Densitom 2013;16:508-19.

26. Cruz-Jentoft AJ, Baeyens JP, Bauer JM, Boirie Y, Cederholm T, Landi F, Martin FC, Michel JP, Rolland Y, Schneider SM, Topinková E, Vandewoude M, Zamboni M; European Working Group on Sarcopenia in Older People. Sarcopenia: European consensus on definition and diagnosis: Report of the European Working Group on Sarcopenia in Older People. Age Ageing 2010;39:412-23.

27. Studenski SA, Peters KW, Alley DE, Cawthon PM, McLean RR, Harris TB, Ferrucci L, Guralnik JM, Fragala MS, Kenny AM, Kiel DP, Kritchevsky SB, Shardell MD, Dam TT, Vassileva MT. The FNIH sarcopenia project: rationale, study description, conference recommendations, and final estimates. J Gerontol A Biol Sci Med Sci 2014;69:547-58.

28. Vitale JA, Bonato M, La Torre A, Banfi G. The Role of the Molecular Clock in Promoting Skeletal Muscle Growth and Protecting against Sarcopenia. Int J Mol Sci 2019;20:4318.

29. Cao L, Morley JE. Sarcopenia Is Recognized as an Independent Condition by an International Classification of Disease, Tenth Revision, Clinical Modification (ICD10-CM) Code. J Am Med Dir Assoc 2016;17:675-7.

30. Sconfienza LM. Sarcopenia: ultrasound today, smartphones tomorrow? Eur Radiol 2019;29:1-2.

31. Ethgen O, Beaudart C, Buckinx F, Bruyère O, Reginster JY. The Future Prevalence of Sarcopenia in Europe: A Claim for Public Health Action. Calcif Tissue Int 2017;100:229-34.

32. Vezzoli A, Mrakic-Sposta S, Montorsi M, Porcelli S, Vago P, Cereda F, Longo S, Maggio M, Narici M. Moderate Intensity Resistive Training Reduces Oxidative Stress and Improves Muscle Mass and Function in Older Individuals.
Antioxidants (Basel, Switzerland) 2019;8:E431.

33. Antunes AC, Araújo DA, Veríssimo MT, Amaral TF. Sarcopenia and hospitalisation costs in older adults: a cross-sectional study. Nutr Diet 2017;74:46-50.

34. Guerri S, Mercatelli D, Aparisi Gómez MP, Napoli A, Battista G, Guglielmi G, Bazzocchi A. Quantitative imaging techniques for the assessment of osteoporosis and sarcopenia. Quant Imaging Med Surg 2018;8:60-85.

35. Onuma T, Kamishima T, Shimamura T, Kawamura N, Yamashita K, Sutherland K, Takeda H. Longitudinal CT study of sarcopenia due to hepatic failure after living donor liver transplantation. Quant Imaging Med Surg 2018;8:25-31.

36. Buckinx F, Landi F, Cesari M, Fielding RA, Visser M, Engelke K, Maggi S, Dennison E, Al-Daghri NM, Allepaerts S, Bauer J, Bautmans I, Brandi ML, Bruyère O, Cederholm T, Cerreta F, Cherubini A, Cooper C, CruzJentoft A, McCloskey E, Dawson-Hughes B, Kaufman JM, Laslop A, Petermans J, Reginster JY, Rizzoli R, Robinson S, Rolland Y, Rueda R, Vellas B, Kanis JA. Pitfalls in the measurement of muscle mass: a need for a reference standard. J Cachexia Sarcopenia Muscle 2018;9:269-78.

37. Kim KM, Jang HC, Lim S. Differences among skeletal muscle mass indices derived from height-, weight-, and body mass index-adjusted models in assessing sarcopenia. Korean J Intern Med 2016;31:643-50.

38. Harvey NC, Kanis JA, Liu E, Johansson H, Lorentzon M, McCloskey E. Appendicular lean mass and fracture risk assessment: implications for FRAX® and sarcopenia. Osteoporos Int 2019;30:537-9.

39. Harvey NC, Odén A, Orwoll E, Lapidus J, Kwok T, Karlsson MK, Rosengren BE, Ribom E, Cooper C, Cawthon PM, Kanis JA, Ohlsson C, Mellström D, Johansson H, McCloskey E. Measures of Physical Performance and Muscle Strength as Predictors of Fracture Risk Independent of FRAX, Falls, and aBMD: A Meta-Analysis of the Osteoporotic Fractures in Men (MrOS) Study. J Bone Miner Res 2018;33:2150-7.

40. Messina C, Piodi LP, Rinaudo L, Emili I, Porro F, Buonomenna C, Sconfienza LM, Vergani L, Ulivieri FM. Bone strain index reproducibility and soft tissue thickness influence: a dual x-ray photon absorptiometry phantom study. Eur Radiol Exp 2019;3:33.

41. Masanés F, Rojano I Luque X, Salvà A, Serra-Rexach JA, Artaza I, Formiga F, Cuesta F, López Soto A, Ruiz D, Cruz-Jentoft AJ. Cut-off Points for Muscle Mass - Not Grip Strength or Gait Speed - Determine Variations in Sarcopenia Prevalence. J Nutr Health Aging 
2017;21:825-9.

42. Kelly TL, Wilson KE, Heymsfield SB. Dual energy X-Ray absorptiometry body composition reference values from NHANES. Vella A, editor. PLoS One 2009;4:e7038.

43. Clinical Guidelines on the Identification, Evaluation, and Treatment of Overweight and Obesity in Adults--The Evidence Report. National Institutes of Health. Obes Res 1998;6 Suppl 2:51S-209S.

44. Romero-Corral A, Montori VM, Somers VK, Korinek J, Thomas RJ, Allison TG, Mookadam F, Lopez-Jimenez F. Association of bodyweight with total mortality and with cardiovascular events in coronary artery disease: a systematic review of cohort studies. Lancet 2006;368:666-78.

45. Kim JY, Han SH, Yang BM. Implication of high-bodyfat percentage on cardiometabolic risk in middle-aged, healthy, normal-weight adults. Obesity (Silver Spring) 2013;21:1571-7.

46. Vasan SK, Osmond C, Canoy D, Christodoulides C, Neville MJ, Di Gravio C, Fall CHD, Karpe F. Comparison of regional fat measurements by dual-energy X-ray absorptiometry and conventional anthropometry and their association with markers of diabetes and cardiovascular disease risk. Int J Obes (Lond) 2018;42:850-7.

47. Wiklund P, Toss F, Weinehall L, Hallmans G, Franks PW, Nordström A, Nordström P. Abdominal and gynoid fat mass are associated with cardiovascular risk factors in men and women. J Clin Endocrinol Metab 2008;93:4360-6.

48. Niederauer CM, Binkley TL, Specker BL. Effect of truncal adiposity on plasma lipid and lipoprotein concentrations. J Nutr Health Aging 2006;10:154-60.

49. Aucouturier J, Meyer M, Thivel D, Taillardat M, Duché P. Effect of Android to Gynoid Fat Ratio on Insulin Resistance in Obese Youth. Arch Pediatr Adolesc Med 2009; 163:826.

50. Bauer JM, Cruz-Jentoft AJ, Fielding RA, Kanis JA, Reginster JY, Bruyère O, Cesari M, Chapurlat R, Al-

Cite this article as: Messina C, Albano D, Gitto S, Tofanelli L, Bazzocchi A, Ulivieri FM, Guglielmi G, Sconfienza LM. Body composition with dual energy X-ray absorptiometry: from basics to new tools. Quant Imaging Med Surg 2020;10(8):16871698. doi: 10.21037/qims.2020.03.02
Daghri N, Dennison E, Kaufman JM, Landi F, Laslop A, Locquet M, Maggi S, McCloskey E, Perna S, Rizzoli R, Rolland Y, Rondanelli M, Szulc P, Vellas B, Vlaskovska M, Cooper C. Is There Enough Evidence for Osteosarcopenic Obesity as a Distinct Entity? A Critical Literature Review. Calcif Tissue Int 2019;105:109-24.

51. de Waal R, Cohen K, Maartens G. Systematic review of antiretroviral-associated lipodystrophy: lipoatrophy, but not central fat gain, is an antiretroviral adverse drug reaction. Darlix J-LEPH, editor. PLoS One 2013;8:e63623.

52. Alikhani A, Morin H, Matte S, Alikhani P, Tremblay C, Durand $M$. Association between lipodystrophy and length of exposure to ARTs in adult HIV-1 infected patients in Montreal. BMC Infect Dis 2019;19:820.

53. Micklesfield LK, Goedecke JH, Punyanitya M, Wilson KE, Kelly TL. Dual-energy X-ray performs as well as clinical computed tomography for the measurement of visceral fat. Obesity (Silver Spring) 2012;20:1109-14.

54. Kuk JL, Katzmarzyk PT, Nichaman MZ, Church TS, Blair SN, Ross R. Visceral Fat Is an Independent Predictor of All-cause Mortality in Men. Obesity (Silver Spring) 2006;14:336-41.

55. Brochu M, Tchernof A, Turner AN, Ades PA, Poehlman ET. Is there a threshold of visceral fat loss that improves the metabolic profile in obese postmenopausal women? Metabolism 2003;52:599-604.

56. Pickhardt PJ, Jee Y, O'Connor SD, del Rio AM. Visceral adiposity and hepatic steatosis at abdominal CT: association with the metabolic syndrome. AJR Am J Roentgenol 2012;198:1100-7.

57. Nicklas BJ, Penninx BWJH, Ryan AS, Berman DM, Lynch NA, Dennis KE. Visceral adipose tissue cutoffs associated with metabolic risk factors for coronary heart disease in women. Diabetes Care 2003;26:1413-20. 